\title{
CONSTRUCTIVE ASPECTS OF NOETHERIAN RINGS ${ }^{1}$
}

\author{
FRED RICHMAN
}

\begin{abstract}
If $R$ is a discrete Noetherian ring, in the sense of Tennenbaum, such that finitely generated ideals are finitely related and detachable, then so is $R[x]$. This shows that a large class of rings enjoy the property that finitely generated ideals are detachable, and that intersections and quotients of finitely generated ideals are finitely generated.
\end{abstract}

1. What is Noetherian? The idea of a commutative Noetherian ring grows naturally out of the study of polynomial rings over fields, and of rings of integers in algebraic number fields. There are many classical characterizations of the Noether condition, the two most common being the following:

Every ideal is finitely generated.

(2) Every ascending chain of ideals is eventually constant.

From a constructive point of view condition (1) is too much to ask for. To understand why, let $I_{n}$ be the set of integers consisting of 0 together with the multiples of the least positive integer $k \leqq n$, if any, such that the sequence 0123456789 occurs in the first $k$ digits of the decimal expansion of $\pi$. Then $I=\bigcup I_{n}$ is an ideal in the ring of integers, and we can even check any integer to see if it is in $I$, but we are currently unable to exhibit a finite set of generators for I. Similarly, condition (2) is a bit too strong but becomes more plausible if we restrict ourselves to finitely generated ideals, which would be silly in condition (1) but is classically equivalent in condition (2). Finding the point of eventual stabilization is still too much to hope for, as can be seen from the sequence of ideals $I_{n}$, so we might content ourselves with just finding two ideals that are equal. Tennenbaum [1] refines this idea and proposes the following definition:

There exists an operation $\rho$ taking finite sequences of elements of $R$ into $R$ such that $\rho\left(a_{1}, \cdots, a_{n}\right)-a_{n}$ is in the ideal generated by $a_{1}, \cdots, a_{n-1}$, and, given any infinite sequence $\left\{a_{i}\right\}$, there exists an $n$ such that $\rho\left(a_{1}, \cdots, a_{n}\right)=0$.

Received by the editors September 4, 1973.

AMS (MOS) subject classifications (1970). Primary 02E99, 13 E05.

${ }^{1}$ This research was supported by NSF Grant GP-28379.

(c) American Mathematical Society 1974 
We call such a ring a Tennenbaum ring. By replacing $R$ by an $R$-module $M$ in condition T, we get the notion of a Tennenbaum module. It turns out [1] that, given an ascending chain of finitely generated submodules of a Tennenbaum module, we can find two equal submodules.

Tennenbaum [1] proves that if $R$ is a Tennenbaum ring, then so is $R[x]$, the ring of polynomials with coefficients in $R$. This is a constructive version of the Hilbert basis theorem. He also shows that a direct sum of Tennenbaum modules is again Tennenbaum. Thus if $R$ is Tennenbaum, then so are finite rank free $R$-modules. We will make use of both these results.

We shall consider only discrete rings. These are rings such that, given two elements $a$ and $b$, we can decide whether $a=b$ or $a \neq b$. Indeed the results of Tennenbaum that we have quoted apply only to such rings.

One difficulty with relying solely on condition (2) to capture the constructive meaning of Noetherian is that quite often you want certain ideals to be finitely generated. For example, if $I$ and $J$ are finitely generated ideals in a Noetherian domain $R$ you might like to lay your hands on a finite set of generators for $I I^{-1}$ or $I \cap J$ or $I: J$. You can certainly do this if $R$ is the ring of integers. You might even want to find a set of generators for the kernel of a homomorphism between two finite rank free $R$-modules, which you can also do, by diagonalizing a matrix, if $R$ is the ring of integers.

Another highly desirable, although apparently unrelated, property is that finitely generated ideals be detachable, that is, you can decide whether a given element of the ring is in a given finitely generated ideal. To establish this property for a large class of rings, one is led to study polynomial rings. It then becomes apparent that a useful condition to have on the ring of coefficients is for finitely generated ideals to be finitely related. This condition also allows us to find the generators mentioned above (Lemma 3), and so becomes a central theme.

2. Finitely related modules. A module $M$ is finitely related if there exists a map $f$ from a finite rank free module onto $M$ such that $\operatorname{ker} f$ is finitely generated. Thus $M$ may be specified by a finite number of generators and a finite number of relations. The following two lemmas are well known but proofs are included to insure that there are no problems from the constructive point of view. The first says that any finite family of generators of a finitely related module is as good as any other.

LEMMA 1. If $M$ is finitely related, then any map of a finite rank free module onto $M$ has a finitely generated kernel.

Proof. Let $f$ and $g$ be maps of finite rank free modules $F$ and $G$ onto $M$. We shall show that if $\operatorname{ker} f$ is finitely generated, then so is ker $g$. Map 
$G \oplus \operatorname{ker} f$ onto $M$ by $g \oplus 0$. Since $G$ is free and $f$ is onto we can write $g=f h$ for some map $h$ from $G$ to $F$. If we extend $h$ to $G \oplus \operatorname{ker} f$ by letting $h$ be the identity on $\operatorname{ker} f$, then $h$ is onto $F$ and $f h=g \oplus 0$ on $G \oplus \operatorname{ker} f$ (see diagram).

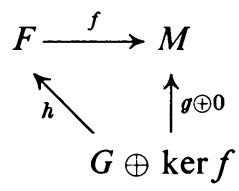

Since $h$ is onto we can write $G \oplus \operatorname{ker} f=G^{\prime} \oplus F^{\prime}$ where $h G^{\prime}=0$ and $h$ takes $F^{\prime}$ isomorphically onto $F$. Then $\operatorname{ker} g \oplus \operatorname{ker} f=\operatorname{ker}(g \oplus 0)=G^{\prime} \oplus K^{\prime}$ where $K^{\prime} \subseteq F^{\prime}$ and $h K^{\prime}=\operatorname{ker} f$. But $G^{\prime}$, being a summand of $G \oplus \operatorname{ker} f$, is finitely generated, and $K^{\prime}$, being isomorphic to $\operatorname{ker} f$, is also finitely generated. Hence ker $g$ is finitely generated, being a summand of $G^{\prime} \oplus K^{\prime}$.

Let $\mathscr{M}$ be the class of modules $M$ such that any map $f$ of a finite rank free module into $M$ has a finitely generated kernel. Note that replacing "finite rank free" by "finitely generated" yields an equivalent statement. The class $\mathscr{M}$ consists of those modules such that every finitely generated submodule is finitely related.

Lemma 2. If $A$ and $B / A$ are in $\mathscr{M}$, then $B$ is in $\mathscr{M}$.

Proof. Denote by $p$ the natural map from $B$ to $B / A$. Suppose $f$ is a map from a finitely generated module into $B$. Then ker $p f$ is finitely generated since $B / A$ is in $\mathscr{M}$. Hence the kernel of the map induced by $f$ from ker $p f$ to $A$ is finitely generated since $A$ is in $\mathscr{M}$. But this last module is just $\operatorname{ker} f$.

The next two lemmas are important from the constructive point of view. Recall that if $B$ and $C$ are submodules of an $R$-module, then $B: C$ consists of those elements $r$ in $R$ such that $r C \subseteq B$.

LEMMA 3. Let $A$ be an element of $\mathscr{M}$. If $B$ and $C$ are finitely generated submodules of $A$, then $B \cap C$ and $B: C$ are finitely generated.

Proof. Let $f$ and $g$ be maps of finite rank free modules $F$ and $G$ onto $B$ and $C$. Then $f-g$ maps $F \oplus G$ into $A$, so $\operatorname{ker}(f-g)$ is finitely generated. Hence $g \pi_{G}(\operatorname{ker}(f-g))=B \cap C$ is finitely generated, where $\pi_{G}$ is the projection on $G$. To show that $B: C$ is finitely generated it suffices, by what we have just proved, to assume that $C$ is cyclic. Then $G$ can be taken to be the ring $R$, and $\pi_{G}(\operatorname{ker}(f-g))=B: C$ is finitely generated.

A subset $A$ of a set $B$ is said to be detachable if, given an element of $B$, you can decide whether or not it is in $A$.

Lemma 4. Let $A$ and $B / A$ be in $\mathscr{M}$ and suppose that $A$ is detachable ( from $B$ ) and that finitely generated submodules of $A$ and $B / A$ are detachable. Then finitely generated submodules of $B$ are detachable. 
Proof. Suppose $C$ is a finitely generated submodule of $B$ and $b \in B$. If $b \notin C(\bmod A)$, then $b \notin C$. If $b \in C(\bmod A)$, then $b-c \in A$ for some $c \in C$. Hence $b \in C$ if and only if $b-c \in C \cap A$. But $C \cap A=\operatorname{ker} f$, where $f: C \rightarrow B \mid A$, so $C \cap A$ is finitely generated and hence $C$ is detachable.

Observe that a consequence of these lemmas is that if $R$ has the property that finitely generated ideals are finitely related (and detachable), then the same is true for finitely generated submodules of free $R$-modules.

3. The basis theorem revisited. Let $P$ be a property of a ring that is classically equivalent to the ring's being Noetherian. Suppose we show, constructively, that if $R$ has property $P$, then so does $R[x]$. We are then legitimately entitled to claim that we have proved a constructive version of Hilbert's basis theorem. The usefulness of such a theorem will result from the balance between the strength of $P$ and the possibility of verifying $P$. As Tennenbaum points out, you can even take $P$ to be condition (1), a condition so strong as to admit no constructive examples, and prove the basis theorem. The condition we suggest adding to the notion of a Tennenbaum ring is that finitely generated ideals be finitely related and detachable. The justification is that this is a useful condition that the integers satisfy and that is inherited under many constructions. In particular it is inherited by $R[x]$ from $R$, which is our main result. First a technical lemma regarding the structure of finitely generated ideals in $R[x]$.

Lemma 5. Let $R$ be a discrete Tennenbaum ring such that finitely generated ideals are finitely related. Let I be a finitely generated ideal in $R[x]$. Then there exists a finitely generated $R$-submodule $M$ of $R[x]$, and an integer $n$, such that

(1) $M$ generates I as an ideal in $R[x]$,

(2) if $f \in M$, then $\operatorname{deg} f \leqq n$,

(3) if $f \in M$ and $\operatorname{deg} f<n$, then $x f \in M$.

Proof. Let $M_{1}$ be an $R$-submodule of $R[x]$ generated by a finite set of generators of $I$. Let $n$ be the maximum of the degrees of elements of $M_{1}$. Given $M_{j}$ we construct $M_{j+1}$ as follows. The elements of $M_{j}$ of degree strictly less than $n$ form a finitely generated $R$-module $N_{j}$, being the intersection of two finitely generated submodules of a finite rank free $R$ module. Set $M_{j+1}=M_{j}+x N_{j}$. Then $M_{j+1}$ is also a finitely generated $R$ module that generates $I$ as an ideal in $R[x]$, and consists of polynomials of degree not exceeding $n$. The chain $M_{1} \subseteq M_{2} \subseteq \cdots$ sits inside a free $R$-module of rank $n+1$. Hence, since $R$ is Tennenbaum, we have $M_{j+1}=M_{j}$ for some $j$. Let $M=M_{j}$. Note that $M=\{F \in I: \operatorname{deg} F \leqq n\}$.

THEOREM. Let $R$ be a discrete Tennenbaum ring such that finitely generated ideals are finitely related (and detachable). Then the same holds for $R[x]$. 
Proof. That $R[x]$ is a discrete Tennenbaum ring is proved in [1]. Let $I$ be a finitely generated ideal in $R[x]$, and let $M$ be the finitely generated $R$ submodule of $R[x]$ constructed in Lemma 5 . To show that $I$ is finitely related, let $S$ be a finite set of generators of $M$, including generators for $\{a \in M: \operatorname{deg} a<n\}$, such that if $s \in S$ and $\operatorname{deg} s<n$, then $x s \in S$. This last condition, together with the generators of the $R$-module relations on $S$, gives a finite set of relations $\mathscr{R}$ on $S$. Now $S$ generates the $R$-module $M$ and hence the ideal $I$. We shall show that any relation $\sum_{s \in S} r_{s} s=0$, where $r_{s} \in R[x]$ is a consequence of the relations in $\mathscr{R}$. We proceed by induction on $m=\max \operatorname{deg} r_{s}$. If $\operatorname{deg} s<n$ and $\operatorname{deg} r_{s}>0$ for some $s$ in $S$, we can use the relations in $\mathscr{R}$ involving multiplication by $x$ to arrange that $\operatorname{deg} r_{s} \leqq 0$ without disturbing the other $r_{t}$ for $\operatorname{deg} t \leqq \operatorname{deg} s$, and without increasing $m$. Hence we may assume that $\operatorname{deg} s<n$ only if $\operatorname{deg} r_{s} \leqq 0$. If $m \leqq 0$, then $r_{s} \in R$ for all $s$ in $S$ so $\sum r_{s} s=0$ is a consequence of $\mathscr{R}$. If $m>0$, let $u_{s}$ be the coefficient of $x^{m}$ in $r_{s}$. Then $\sum u_{s} s$ has degree strictly less than $n$ so there are elements $v_{s}$ in $R$ such that $\sum u_{s} s=\sum v_{s} s$, and $v_{s}=0$ if deg $s=n$. But this is a consequence of $\mathscr{R}$, so we can use $\mathscr{R}$ to decrease $m$, and we are done by induction.

Suppose, in addition, that finitely generated ideals of $R$ are detachable. Clearly $I=M+x M+x^{2} M+\cdots$. By induction it is easily seen that if $f \in$ $\sum_{i=0}^{k} x^{i} M$ and $\operatorname{deg} f<n+k$, then $x f \in \sum_{i=0}^{k} x^{i} M$. Now suppose we are given $f$ in $R[x]$ such that $\operatorname{deg} f \leqq n+s$. Then $f \in \sum_{i=0}^{m} x^{i} M$ for some $m \geqq s$ so $x^{m-s} f \in \sum_{i=0}^{m} x^{i} M$. Thus

$$
x^{m-s} f \in \sum_{i=m-s}^{m} x^{i} M=x^{m-s} \sum_{i=0}^{s} x^{i} M
$$

whereupon $f \in \sum_{i=0}^{s} x^{i} M$. So to check if $f$ is in $I$, we check to see if $f$ is in the finitely generated, and hence detachable, $R$-submodule $\sum_{i=0}^{s} x^{i} M$.

Note that one corollary to this theorem is that finitely generated ideals in the polynomial ring $Z\left[x_{1}, \cdots, x_{n}\right]$ are finitely related and detachable, answering a question of Tennenbaum. Moreover this property is clearly preserved under the taking of finite products. If we take the quotient of such a ring modulo a finitely generated ideal, we certainly get a discrete Tennenbaum ring. It remains to show that we get a ring in which finitely generated ideals are finitely related (they are clearly detachable) to establish a conjecture of Tennenbaum that finitely generated ideals are detachable in any ring gotten from the integers by forming polynomial rings, quotient fields, finite products, and dividing out by finitely generated ideals.

Proposition. Let $R$ be a ring such that finitely generated ideals are finitely related, and let I be a finitely generated ideal of $R$. Then finitely generated ideals of $R / I$ are finitely related. 
Proof. Let $J / I$ be a finitely generated ideal in $R / I$. Then $J$ is a finitely generated ideal in $R$. Let $f$ and $g$ map finite rank free $R$-modules $F$ and $G$ onto $J$ and $I$. Then $f-g$ maps $F \oplus G$ onto $J \oplus I$ with finitely generated kernel $K$. Now $f$ induces a map of the finite rank free $R / I$-module $F / I F$ onto $J / I$. The kernel of this map is readily seen to be $\left(\pi_{F} K\right) / I F$ which is finitely generated.

One last remark. If $R$ is a ring in which finitely generated ideals are detachable, then condition $T$ can be simplified to read

$\left(\mathrm{T}^{\prime}\right)$ Given any infinite sequence $\left\{a_{i}\right\}$ of elements of $R$, there exists an $n$ such that $a_{n}$ is in the ideal generated by $a_{1}, \cdots, a_{n-1}$.

In fact we simply set $\rho\left(a_{1}, \cdots, a_{n}\right)$ equal to 0 , or to $a_{n}$, depending on whether $a_{n}$ is in the ideal generated by $a_{1}, \cdots, a_{n-1}$ or not.

\section{REFERENCE}

1. J. B. Tennenbaum, A constructive version of Hilbert's basis theorem, Ph.D. Dissertation, University of California, San Diego, Calif., 1973.

Department of Mathematics, New Mexico State University, Las Cruces, New MeXico 88003 